\title{
Cuidar de Imigrantes: das interaçóes em contexto à construçáo de competências culturais nos enfermeiros
}

Caring for Immigrants: from interacting in practice to building nurses' cultural competencies Atender a los inmigrantes: de las interacciones en el contexto a la construcción de competencias culturales en enfermeros

Alcinda Sacramento Costa dos Reis*; Maria Arminda Mendes Costa**

\section{Resumo}

Enquadramento: Cuidar de imigrantes proporciona aos enfermeiros uma nova forma de olhar sobre si mesmos e sobre aqueles de quem cuidam. As interações entre estes atores estão na base do desenvolvimento das competências culturais nos enfermeiros e da vivência equilibrada de processos de transição nos imigrantes, de acordo com Leininger e Meleis.

Objetivos: Estudar o processo de construção das competências culturais nos enfermeiros, partindo da identificação dos significados atribuídos nas díades em contexto de cuidados (unidades de saúde familiar e domicílios dos imigrantes).

Metodologia: Este estudo qualitativo e etnográfico mobilizou a presença do investigador com os atores no contexto. Utilizámos na recolha de dados: narrativas, observação participante, entrevistas etnobiográficas e grupos de discussão. Participantes selecionados: integrantes de unidades de saúde e voluntários para o estudo - 23 enfermeiros, 27 imigrantes e 2 informantes privilegiados (mediadora cultural e médico). Resultados: As competências culturais nos enfermeiros constroem-se de forma processual no contexto de cuidados. O processo iniciase nas interações com os imigrantes; os enfermeiros identificam as áreas deficitárias nos conhecimentos e habilidades culturais; e tomam consciência do património cultural bilateral nos encontros.

Conclusão: A consciência cultural, associada à motivação para cuidar de imigrantes, assenta numa base evolutiva do etnocentrismo para o relativismo étnico na prática clínica dos enfermeiros.

Palavras-chave: competência cultural; enfermeiros; emigrantes e imigrantes.

\begin{abstract}
Background: Immigrant care provides nurses with a new way to look at themselves and those they care for. According to Leininger and Meleis, the interaction between them underlies the development of cultural competencies in nurses and a balanced experience of transition processes in immigrants.

Objectives: To study the process of building cultural competencies in nurses based on the identification of the meanings assigned in dyads in health care settings (family health units and immigrants' households). Methodology: This qualitative and ethnographic study mobilised the researcher to be present in loco with the participants. Data were collected using narratives, participant observation, ethno-biographical interviews and focus groups with a group of volunteers selected from health care units: 23 nurses, 27 immigrants and 2 privileged informants (a cultural mediator and a physician).

Results: In health care contexts, nurses' cultural competencies are built in a procedural way. This process begins with their interaction with immigrants; nurses then identify deficit areas in their cultural knowledge and skills; and recognise the bilateral cultural heritage during those meetings.

Conclusion: In nurses' clinical practice, cultural awareness, combined with the motivation to take care of immigrants, is based on an evolutionary basis, from ethnocentrism to ethnic relativism.
\end{abstract}

Keywords: cultural competency; nurses; emigrants and immigrants.

\footnotetext{
* Doutoranda em Ciências de Enfermagem - ICBAS/Universidade do Porto. Mestre em Ciências de Enfermagem. Licenciatura em Enfermagem na Comunidade. Professora Adjunta. Instituto Politécnico de Santarém - Escola Superior de Saúde de Santarém 2005-075, Santarém, Portugal [alcinda.reis22@gmail.com]. Morada para correspondência: Rua Rádio Ribatejo, 1, $5^{\circ}$ Dto, 2000-219 Santarém - Portugal

** Professora Coordenadora com Agregação da Escola Superior de Enfermagem do Porto, 4200-072, Porto, Portugal [armindamcosta@gmail.com].
}

\section{Resumen}

Marco contextual: La atención a inmigrantes proporciona a los enfermeros una nueva forma de verse a sí mismos y a los que cuidan. Las interacciones entre estos actores están en la base del desarrollo de competencias culturales en los enfermeros y de la experiencia equilibrada de procesos de transición en los inmigrantes, de acuerdo con Leininger y Meleis.

Objetivos: Estudiar el proceso de construcción de las competencias culturales en los enfermeros a partir de la identificación de significados asignados en las díadas, en contextos de atención (unidades de salud familiar y hogares de los inmigrantes).

Metodología: Este estudio cualitativo y etnográfico permitió que el investigador estuviese con los actores en el contexto. En la recogida de datos, se utilizaron: narrativas, observación participante, entrevistas etnobiográficas y grupos de discusión. Participantes seleccionados: integrantes de unidades de salud y voluntarios - 23 enfermeros, 27 inmigrantes y 2 informantes privilegiados (mediadora cultural y médico). Resultados: Las competencias culturales en los enfermeros se construyen de forma procesual en los contextos de atención de enfermería. Este proceso comienza en las interacciones con los inmigrantes; los enfermeros identifican las áreas deficitarias relativas a los conocimientos y las habilidades culturales, y toman conciencia del patrimonio cultural bilateral en los encuentros.

Conclusión: La conciencia cultural, asociada a la motivación para atender a los inmigrantes, se basa en una evolución del etnocentrismo al relativismo étnico en la práctica clínica del personal de enfermería.

Palabras clave: competencia cultural; enfermeros; emigrantes e inmigrantes.

Recebido para publicação em: 16.05 .13

Aceite para publicação em: 21.01.14 


\section{Introdução}

Os contextos de cuidados de saúde são hoje caracterizados por diversidades étnicas e culturais sendo que os sujeitos de cuidados em Enfermagem são frequentemente imigrantes de diferentes origens, vivenciando as inerentes fases de transição (Meleis, 2010). As interações estabelecidas nos contextos de cuidados entre os enfermeiros e os imigrantes e as competências culturais desenvolvidas a partir delas, constituem-se como os pólos organizadores deste estudo. Segundo o Relatório de Imigração, Fronteiras e Asilo do Serviço de Estrangeiros e Fronteiras (Serviço de Estrangeiros e Fronteiras. Departamento de Planeamento e Formação. Núcleo de Planeamento, 2012), o fluxo migratório para Portugal em 2011 registou um decréscimo de 10,6\% face ao ano de 2010. Continua a evidenciar-se, contudo, a relevância destes cidadãos enquanto consumidores de cuidados de saúde, e especificamente de Enfermagem, com as suas particularidades culturais em contexto de cuidados. Para este estudo adotámos o conceito de imigrantes que os define como todos aqueles que sendo estrangeiros "são detentores de título de residência e os estrangeiros a quem foi prorrogada a permanência de longa duração" (Serviço de Estrangeiros e Fronteiras. Departamento de Planeamento e Formação. Núcleo de Planeamento, 2012, p.15).

Concordando com a perspetiva apontada pelo Alto Comissariado para a Saúde em Portugal, enfatizamos a importância atribuída à saúde e aos cuidados aos imigrantes, colocando-se neste campo duas questões essenciais: a importância de se assegurarem os desafios que a saúde dos imigrantes coloca dentro das fronteiras do território nacional e a definição de estratégias que assegurem a saúde das pessoas na sua diversidade cultural, como apontam Machado, Pereira, e Machaqueiro (2010), sendo que estas dimensões vêm também suportar o interesse do nosso estudo.

Para a Ordem dos Enfermeiros, no que se refere ao exercício profissional e às suas competências em cuidados gerais, podemos perceber a imprescindibilidade apontada de que os enfermeiros tenham sensibilidade para lidar com as diferenças culturais na interação com as pessoas na sua prática clínica (Ordem dos Enfermeiros. Conselho de Enfermagem, 2003).

Tal como no estudo de Serrano, Costa, e Costa (2011), valorizamos a importância dos processos de interação dos enfermeiros com os sujeitos de cuidados, a par de uma aprendizagem experiencial desenvolvida e da construção de competências produzidas, sendo que a nossa investigação se focou no desenvolvimento das competências culturais.

Vários autores vêm enfatizando a importância da dimensão cultural nos processos de interação e cuidados a imigrantes, como no caso de estudos nacionais desenvolvidos por Anes (2006) e Pereira (2008) com objetos de estudo e perspetivas de análise transversais aos cuidados desenvolvidos pelas equipas de saúde; e outros internacionais, como o estudo de Skott e Lundgren (2009), que especifica as interações em contextos multiculturais de cuidados desenvolvidos por enfermeiros. Propusemo-nos a estudar o processo da construção das competências culturais nos enfermeiros, partindo da identificação de significados atribuídos nas díades em contexto de cuidados, especificamente em Unidades de Saúde Familiar e nos domicílios dos imigrantes. Nesta base reflexiva, questionámos: como se desenvolve o processo de construção das competências culturais nos enfermeiros para cuidar imigrantes? Visando a clarificação do problema colocado, procedemos à triangulação metodológica, de atores e de contextos em sustentação das elaborações interpretativas que pretendíamos e em consonância com a orientação etnográfica do estudo.

\section{Enquadramento}

\section{Um referencial na prática clínica com imigrantes}

Entendemos a perspetiva teórica que elaborámos como "versões ou perspetivas, através das quais se vê a realidade" (Flick, 2005, p.44), portanto concordantes com o processo de descoberta da investigação qualitativa que desenvolvemos.

Pensarmos na multiculturalidade em Enfermagem é também pensarmos no lugar, na visibilidade e no reconhecimento que as competências culturais têm tido no desenvolvimento da prática clínica, e também, inversamente, na forma como esta tem contribuído para as competências culturais dos enfermeiros na atualidade, como referenciam diferentes autores (Campinha-Bacote, 2011; Purnell, 2011). Estudando o processo de construção das competências culturais 
nos enfermeiros, colocámo-nos numa perspetiva bidimensional de problematização por via dos diferentes atores envolvidos: uns - os enfermeiros, porque são responsáveis pelo planeamento, desenvolvimento e avaliação dos cuidados, e os outros - os imigrantes, porque são o foco da atenção profissional dos primeiros; desta forma esperámos que estes sujeitos de cuidados espelhassem a face visível dessas competências dos enfermeiros quando acompanhados nas suas vivências de saúde e de doença.

Selecionámos para a conceptualização do estudo a teoria da diversidade e universalidade do cuidado cultural de Leininger (1994), uma vez que esta propõe a descoberta das diversidades (diferenças) e universalidades (semelhanças) culturais no cuidado humano, tais como as que encontrámos nas díades enfermeiros/imigrantes para a produção de novo conhecimento orientador da prática de Enfermagem. A adoção da perspetiva desta autora permitiu-nos, tal como refere Purnell (2011), obter a compreensão de especificidades culturais nos cuidados ligadas a crenças e condutas associadas aos contextos e definidoras das interações entre quem cuida e quem é cuidado. Valorizámos, de acordo com a teoria de Leininger, a visão holística, distinta da forma fragmentada de olhar para as pessoas e compreensiva em contextos multiculturais de cuidados, em torno do conceito de cultura, referenciado na mesma fonte como "específico padrão de comportamento que distingue qualquer sociedade de outras e dá sentido às expressões humanas do cuidar" (Culture Care Theory: A major contribution to advance transcultural nursing knowledge and practices, 2002, citado em Purnell, 2011, p. 532). Procurámos a mobilização desta perspetiva como suporte ontológico de explicações e interpretações do fenómeno em estudo - construção das competências culturais nos enfermeiros; ainda de acordo com a perspetiva teórica de Leininger, distinguimos cuidados de cuidar: o primeiro conceito implica a provisão dos "necessários e personalizados serviços que ajudam o homem a manter o seu estado de saúde ou a recuperar da doença", e o segundo "usado como verbo implica um sentimento de compaixão, interesse e preocupação pelas pessoas" (1994, p.30).

Recorremos também ao suporte da teoria das transições de Meleis (2010), relativamente à análise das relações humanas e das mudanças - transições, que de acordo com esta autora ocorrem no percurso de vida das pessoas ao longo do tempo em situações e contextos particulares; esta perspetiva teórica sustentou-nos a análise dos processos de saúde e doença dos imigrantes, a quem Meleis se referiu em particular, bem como às suas interações com os enfermeiros em contextos de cuidados. O conceito de transição foi assumido neste estudo de acordo com a perspetiva de Meleis (2010), que considera ser central à prática clínica de Enfermagem e à génese das modificações de vida, saúde, relações e ambiente das pessoas, nas quais os enfermeiros têm assumido um papel relevante no acompanhamento na entrada, na passagem e na saída destas fases. Especificamente em relação ao conceito de transição em imigrantes, considerámos neste estudo, tal como Meleis (2010), que estas experiências nas suas vidas vão muito para além das fronteiras geopolíticas, atravessando fases e condições de vida, sendo contributivas para a sua própria auto-definição como pessoas. Pensámos no que os enfermeiros necessitariam para suplementar estas situações, tal como apontou Meleis (2010) e a que Purnell (2011) se referiu como a necessidade de que os enfermeiros se "percebam como culturalmente competentes" (p. 529).Tal necessidade surgiu também enfatizada no estudo de Vega (2010) que apontou, tal como Leininger o fez no passado (1994), os subsídios da antropologia como suscetíveis de contribuírem para desenvolver nos enfermeiros uma maior capacidade reflexiva sobre si mesmos e a sua prática clínica, particularmente nos cuidados a imigrantes.

Refletimos, na linha de diversos autores, sobre os sujeitos de cuidados serem pessoas com diferentes referências culturais daquelas que são dominantes nos contextos de cuidados do país de acolhimento (Fonseca, Silva, Esteves, \& McGarrigle, 2009; Meleis, 2010). Relevámos a importância da autorreflexão dos próprios valores culturais dos enfermeiros para a construção das suas competências culturais (Campinha-Bacote, 2011; Purnell, 2011); valorizámos também a reflexão das culturas e subculturas do contexto organizacional de saúde para os enfermeiros, em concordância com os estudos de Vega (2010) e de Serrano et al. (2011). Esta valorização decorreu da constatação de que o mais importante para o comportamento e desenvolvimento humanos está muitas vezes ligado à forma como o ambiente é "percebido e não conforme ele poderia existir na 
realidade «objetiva»", como refere Bronfenbrenner (2002, p.6) no modelo ecológico do desenvolvimento humano. Progressivamente foi-se traduzindo para nós e para este estudo, com foco na dimensão da cultura dos atores (enfermeiros e imigrantes) e dos contextos de cuidados, uma necessidade de valorização dinâmica de aquisição de experiências e competências ao longo do percurso profissional em contexto da prática clínica dos enfermeiros, como surge enfatizado no estudo de Serrano et al. (2011). A construção das competências culturais pelos enfermeiros também nos surgiu associada à perspetiva de Campinha-Bacote (2011), que defende que o desenvolvimento dos profissionais implica a construção ativa de semelhanças entre si mesmos e os imigrantes, no contexto dos cuidados, para além da sua necessária identificação, já anteriormente referida por Leininger (1994).

Tal como Campinha-Bacote (2011), mobilizámos como referência para este estudo as competências culturais nos enfermeiros construídas com base na existência de: sensibilidade cultural; auto-consciência, conhecimento; humildade e habilidades culturais; e planeamento das interações com os imigrantes nos designados encontros culturais, partindo da necessária motivação inicial dos enfermeiros.

Nesta linha de pensamento definimos como questões de investigação: Como se caraterizam as interações estabelecidas nos contextos de cuidados entre enfermeiros e imigrantes? Que dificuldades e maisvalias são identificadas em contextos multiculturais?

De acordo com a nossa perspetiva considerámos a influência das interações dos enfermeiros com os imigrantes como promotora de adaptações nas díades de cuidados e como produtora do desenvolvimento de atitudes, motivações e comportamentos para a construção de competências culturais nos enfermeiros.

\section{Metodologia}

De acordo com a perspetiva teórica descrita e em resposta às questões colocadas, considerámos uma lógica indutiva e interpretativa de estudo, valorizando os significados atribuídos pelos atores nos contextos de interação.

Colocado este estudo num paradigma qualitativo e etnográfico de investigação, reconstituímos os significados do processo de construção de competências culturais nos enfermeiros, com um total de 52 participantes voluntários, após solicitação prévia: 23 enfermeiros, uma mediadora cultural e um médico - profissionais das unidades de saúde onde decorreu o estudo; e 27 imigrantes - utentes das mesmas unidades. Os participantes abriram caminho ao investigador através da bola de neve de uns para os outros, à medida que permanecíamos no terreno e que íamos experienciando progressivamente os diferentes papéis de estranho a membro, como apontou Flick (2005). A mobilização da mediadora cultural e do médico, ambos das unidades de saúde, para a recolha de informação proporcionou-nos a garantia da validade interna do estudo pelo domínio conceptual que estes têm sobre o fenómeno, de acordo com o que sugerem Durand e Blais (2003).

\section{Procedimentos de recolha de dados}

Ao definirmos no desenho do estudo os métodos de investigação, visámos a construção de uma corrente lógica de evidência, numa perspetiva recursiva de recolha e análise de dados, tal como apontaram Streubert e Carpenter (2013), que nos permitisse a elaboração de uma heurística compatível com a caraterização cultural das competências dos enfermeiros quando cuidam imigrantes.

Para a recolha dos dados utilizámos os seguintes métodos de investigação etnográfica: análise de narrativas escritas focalizadas na interação e nos cuidados, que solicitámos aos enfermeiros e imigrantes das referidas unidades; observação participante com registo descritivo e posteriormente focalizado (Spradley, 1980) dos cuidados de Enfermagem a imigrantes; e entrevistas etnobiográficas a enfermeiros, a imigrantes e também a informantes privilegiados - (mediadora cultural e médico). O guião das entrevistas foi estruturado a partir da recolha e análise dos dados das narrativas solicitadas e da fase inicial de observação participante, com uma orientação semi-diretiva no seu desenvolvimento (Flick, 2005). Em concordância com Streubert e Carpenter (2013), considerámos que "o uso de múltiplos métodos de colheita de dados é importante pois aumenta a credibilidade dos resultados" (p. 206).

O trabalho de campo ocorreu por períodos intermitentes entre setembro de 2011 e outubro de 2012. Optámos pelo desenvolvimento do estudo em 
cuidados de saúde primários na Região de Saúde de Lisboa e Vale do Tejo, em contextos de cuidados de Enfermagem à população imigrante - Unidades de Saúde Familiar e unidades de cuidados na comunidade em situações de cuidados domiciliários a imigrantes. Esta opção prendeu-se com as possibilidades constatadas de melhor acesso e desenvolvimento da investigação. Considerando o relatório Mighealthnet sobre o estado da arte em Portugal (Fonseca et al., 2009) e a perspetiva de Machado et al. (2010), identificámos que os cuidados de saúde primários são frequentemente a porta de entrada para os imigrantes no Serviço Nacional de Saúde numa primeira fase no país de acolhimento, nomeadamente por via da promoção da saúde e prevenção da doença na área da saúde infantil (dos filhos). Assumimos esta constatação como compatível com a continuidade da adesão aos cuidados destes sujeitos ao longo do estudo e, portanto, tornando possível a acessibilidade e a continuidade da nossa investigação.

Relativamente às preocupações éticas como investigadores, estas prenderam-se especificamente com a proteção dos participantes, sendo que sentimos necessidade de conciliação entre a clareza nos objetivos do estudo e a utilização dos diferentes métodos para a recolha de dados que nos propusemos utilizar, sem que se registassem constrangimentos nos sujeitos estudados nem a perda da espontaneidade desejada na obtenção dos dados. Cientes da delicadeza das situações de observação participante no processo de cuidados em casa dos imigrantes ou nas unidades de saúde e ainda nas situações dos grupos de discussão, comprometemonos a respeitar plenamente o sigilo e a privacidade na recolha de dados em todos os métodos utilizados, bem como o anonimato dos diferentes intervenientes no processo de cuidados. Fizemo-lo recorrendo à clarificação e ao preenchimento do consentimento informado com cada um dos participantes, como propuseram Streubert e Carpenter (2013). Fomos progressivamente acedendo ao conhecimento cultural dos enfermeiros e dos imigrantes, desenvolvendo as necessárias inferências culturais (Streubert e Carpenter, 2013) face à forma como se percecionavam em relação às suas competências culturais (os enfermeiros) e se sentiam mais ou menos culturalmente ajudados e compreendidos nas suas transições de saúde - doença (os imigrantes). Tratou-se de um processo de focalização progressiva à medida que fomos desenvolvendo a análise das narrativas, do corpus das entrevistas etnobiográficas, com centralização no momento-charneira-interações e cuidados entre enfermeiros e imigrantes, e dos dados da observação participante.

$\mathrm{Na}$ fase final do trabalho de campo, devolvemos os dados analisados aos dois tipos de sujeitos, procurando simultaneamente a validação das interpretações feitas e a saturação dos dados, concretizada com as informações redundantes obtidas. Fizemo-lo recorrendo a dois distintos grupos de discussão (um de imigrantes e um de enfermeiros), estimulando respostas a questões gerais, primeiro, e depois, progressivamente, a questões mais focalizadas na problemática em estudo. A estruturação deste planeamento ocorreu após a análise taxonómica e componencial dos dados da observação participante descritiva e focalizada (Spradley, 1980). Os grupos de discussão foram, portanto, orientados com base em guiões que permitiram o registo da produção discursiva dos participantes na sua especificidade, de acordo com a proposta de Geoffrion (2003). $\mathrm{Na}$ linha do mesmo autor, foi-nos possível nestes momentos perceber se havia uma significação comum ou diferente das questões colocadas a um e outro grupos, valorizando as possibilidades de, por um lado, obter resultados rapidamente e, por outro, de utilizar a flexibilidade de uma técnica que permite a inversão e/ou reformulação das questões colocadas. Baseámo-nos na orientação conceptual do modelo de competências culturais de CampinhaBacote (2011) para o desenvolvimento do guião do grupo dos enfermeiros, considerando os constructos: consciência cultural; conhecimento cultural; habilidade cultural; encontro cultural; e desejo cultural. Para o grupo dos imigrantes baseámo-nos nas dimensões consciência, padrões de resposta e perceção, do conceito de transição saúde-doença, apontadas por Meleis (2010). Refletindo sobre o nosso papel na utilização deste tipo de técnica de pesquisa e sabendo que poderíamos facilmente influenciar a produção dos dados no terreno, optámos por calendarizar em primeiro lugar o grupo de discussão dos enfermeiros. Considerámos que neste a nossa presença seria rapidamente conotada com a cultura indígena, favorecendo-nos a apropriação da realidade social destes participantes e a possibilidade de ser posteriormente rentabilizada na condução do grupo com imigrantes, tal como aponta 
Flick (2005). Sabendo da necessidade de que cada sujeito se percebesse na sua cultura em concordância com Purnell (2011), individualmente e em contexto coletivo, e considerando que nos interessava conhecer as especificidades dos diferentes tipos de sujeitos apontados, definimos na perspetiva do mesmo autor e de acordo com a problemática do estudo uma questão base para cada um dos grupos:

- Grupo dos enfermeiros: "Até que ponto se reconhecem como construtores das vossas competências culturais quando cuidam de pessoas imigrantes?"

- Grupo dos imigrantes: "Até que ponto os enfermeiros vos ajudam nos processos de transição saúde - doença?".

\section{Resultados e Discussão}

Foi-nos previamente facilitado o acesso ao campo (Flick, 2005), tal como havia acontecido anteriormente com as respetivas enfermeiras de família dos imigrantes, com uma captação eficaz destes sujeitos para o momento da discussão em grupo que visou a validação e saturação dos dados obtidos a partir das técnicas anteriores.

Baseando-nos nos constructos e dimensões referidas, respetivamente para enfermeiros e para imigrantes, passámos à discussão dos resultados obtidos com os dois grupos de participantes, que apresentámos de acordo com as questões de investigação anteriormente colocadas.

Face à questão "como se caracterizam as interações estabelecidas nos contextos de cuidados entre enfermeiros e imigrantes?", obtivemos:

\section{Nos imigrantes}

Os padróes de resposta identificados nos encontros com enfermeiros

$\mathrm{Na}$ caracterização destes padrões pelos imigrantes surgiu a comparação face aos enfermeiros do país de origem, valorizando-se a relação de proximidade com estes profissionais em Portugal; pela nossa interpretação, esta proximidade é conotada com a individualização sentida na preparação e decurso dos encontros, uma vez que os imigrantes reconheceram no papel dos enfermeiros a contribuição para o seu reequilíbrio e segurança nos processos de transição de saúde-doença. Surgiu ainda a referência ao tratamento privilegiado de que se sentem alvo nos encontros culturais com os enfermeiros, exatamente por estarem na condição de minoria como imigrantes, ao contrário do sentimento de marginalização mais frequentemente apontado por este tipo de atores, como sugerem outros estudos (Skott \& Lundgren, 2009; Vega, 2010).

\section{Nos enfermeiros \\ O planeamento e o decurso dos encontros culturais}

A necessidade de mais tempo para desenvolver uma relação nas díades de cuidados assumiu-se, quer em contexto de Unidades de Saúde Familiar, quer em visita domiciliária aos imigrantes. Identificam a necessidade de serem mais cautelosos na validação da mensagem transmitida e recebida, implicando mais tempo nas interações. Surgiram ainda, enfatizadas pelos enfermeiros situações de constrangimentos nos encontros quando os intérpretes presentes são familiares dos imigrantes (por vezes os filhos), na linha apontada no estudo de Skott e Lundgren (2009), identificámos aqui uma sensação de ambivalência dos enfermeiros na presença de intérpretes (familiares ou não), face à necessidade de, por um lado, poderem recorrer a alguém que faça a tradução mas, por outro, a sua presença se poder tornar constrangedora para a pessoa cuidada.

\section{A manifestaçáo de desejo cultural}

A identificação da motivação para cuidar de imigrantes para os enfermeiros deste estudo surgiu associada à ideia de maior desenvolvimento profissional, referindo que ao saberem cuidar adequadamente de imigrantes, sabem cuidar da individualidade, em qualquer situação, qualquer pessoa; esta postura, na nossa perspetiva, é sobreponível à linha de pensamento de Campinha-Bacote (2011) relativamente às características de flexibilidade e humildade culturais, que a autora salienta como fundamentais para o desenvolvimento de competências culturais nos enfermeiros, aprendendo com os outros e olhandoos como informantes culturais.

Em resposta à questão "que dificuldades e maisvalias são identificadas em contextos multiculturais?", obtivemos: 


\section{Nos imigrantes}

A consciência face à influência dos enfermeiros nos seus processos de saúde-doença Os imigrantes enfatizaram como mais-valia a ideia de que os enfermeiros nas unidades de saúde familiar e quando os visitam em casa, lhes transmitem conhecimentos importantes relativamente à vivência das situações de saúde e de doença, numa lógica semelhante ao que consideram que ocorre com os cidadãos portugueses. Evidencia-se uma necessidade de afirmação de pertença e de simetria relacional que privilegie as universalidades ao invés das diversidades nos encontros, em consonância com o estudo de Vega (2010). Consideraram existir situações de persistência de dúvidas no processo de comunicação, nomeadamente no contexto das consultas de Enfermagem, assumindo de forma pouco explícita o registo pontual de um contributo insuficiente nas suas transições de saúde-doença. Contudo, remeteram a análise desta constatação para a necessidade de se apurarem responsabilidades, que consideraram que deviam ser repartidas por ambos os atores nas díades de cuidados. Consideramos aqui, que a pouca abertura dos imigrantes para assumir de forma explícita as suas dificuldades no processo de comunicação e interação em cuidados se ligou à identificação da nossa sobreposição de papéis, como investigadora e enfermeira, o que se constituiu como uma limitação neste momento do estudo.

\section{A perceçáo dos contributos dos enfermeiros para a sua estabilidade nas transiçóes vivenciadas}

O nível de proximidade nas díades surgiu assumido como uma mais-valia na tomada de decisão dos imigrantes nos processos de saúde-doença individuais e familiares. Este aspeto parece evidenciar-se a par das diferenças que registam e valorizam sobre as competências nos enfermeiros que deles cuidam nos contextos, face às que recordam destes profissionais nos países de origem. O uso do humor pelos enfermeiros foi claramente apontado pelos imigrantes como um elemento facilitador e como sinónimo de segurança nos cuidados aos seus filhos, contribuindo para a perda de medos dos adultos e das crianças.

\section{Nos enfermeiros}

\section{O desenvolvimento da consciência cultural}

Para os enfermeiros os encontros com os imigrantes estimulam o levantamento de questões sobre a forma como olham para dentro de si mesmos, para melhor compreenderem os outros nos seus projetos individuais de saúde ede vida. Valorizama possibilidade de desenvolvimento da capacidade de empatia nestes encontros por tentarem frequentemente colocar-se no lugar do outro. Apontam dificuldades na reflexão sobre a sua prática clínica e nos momentos planeados de discussão em equipa de pares e multidisciplinar. Acreditamos que estas dificuldades surgem relacionadas com o autoexame e a autoexploração propostos por Campinha-Bacote (2011) em ordem ao desenvolvimento de competências culturais nos enfermeiros.

\section{A identificaçáo de áreas de conhecimento cultural}

Para os enfermeiros participantes no estudo, cuidar imigrantes estimula a sua pesquisa sobre o que está na base de comportamentos adotados por estas pessoas. Enfatizam dificuldades em compreender como os imigrantes enquadram culturalmente a saúde e a doença. Esta postura está na nossa perspetiva, de acordo com um dos eixos identificados por Campinha-Bacote (2011), para a focalização dos cuidados culturalmente competentes, baseados no conhecimento de crenças de saúde e de valores dos imigrantes. Por outro lado, também surgiu enfatizado pelos enfermeiros que um dos objetivos quando cuidam de imigrantes é permitir-lhes o entendimento daquilo que lhes pode ser oferecido pelas organizações prestadoras de cuidados de saúde. Esta preocupação vai ao encontro do estudo de Serrano et al. (2011), que enfatiza a importância do desenvolvimento da competência coletiva entre indivíduos, baseado num ambiente de aprendizagem entre eles e a organização.

\section{As habilidades culturais utilizadas em situaçóes de interaçáo}

A caracterização destas habilidades surgiu associada à promoção da segurança e à atenção específica. Esta assunção vai ao encontro da manifestação dos imigrantes no seu grupo de discussão ao referirem que se sentem um foco de especial atenção por serem 
imigrantes. Os enfermeiros identificam dificuldades no planeamento de cuidados individualizados com estas pessoas, na comunicação, ligadas à barreira da língua e ao enquadramento cultural da mensagem. A presença de crianças (filhas dos imigrantes emergiu), todavia, como um elemento facilitador no decurso dos encontros.

\section{Conclusão}

Os enfermeiros consciencializam progressivamente nas díades com imigrantes a sua cultura pessoal, profissional e organizacional, como não sendo a única matriz possível para uma leitura da realidade nas situações de transição de saúde-doença destas pessoas. Esta consciencialização abre aos enfermeiros outras possibilidades de desenvolvimento profissional, no sentido da evolução de uma visão da realidade baseada no etnocentrismo para uma outra mais relativa, em que a cultura própria dos profissionais se vai constituindo apenas como organizadora, entre múltiplas outras possibilidades que vão conseguindo identificar nas pessoas de quem cuidam. Este processo decorre à medida que as suas competências culturais se vão construindo.

As interações entre enfermeiros e imigrantes no estudo identificam-se pelas caraterísticas apresentadas, em resposta à primeira questão de investigação que colocámos.

No que concerne a dificuldades e mais-valias nos contextos multiculturais estudados, sobre as quais também nos questionámos, concluímos que a identificação e pesquisa ativas pelos enfermeiros de áreas deficitárias nos seus conhecimentos e habilidades para cuidar imigrantes são valorizadas pelos profissionais para a construção das suas competências culturais. A presença de crianças (filhas dos imigrantes) surge particularmente identificada como uma mais-valia para os dois tipos de participantes, apresentando-se como um elemento facilitador no decurso dos encontros, em ordem à coerência nos cuidados. Os enfermeiros identificam nesta presença, uma probabilidade aumentada de interagir mais rápido e mais facilmente com os respetivos pais na sua prática clínica, emergindo aqui para nós uma importante linha de investigação. A simetria relacional nas díades, a proximidade e a flexibilidade nas interações estabelecidas, emergem também com um relevante significado atribuído pelos atores neste estudo, por vezes associado a fonte de dificuldades.

Registámos a importância dos dados apresentados na construção das competências culturais nos enfermeiros bem como na produção da harmonia e de um mais rápido equilíbrio nas transições de saúdedoença dos imigrantes.

Tendo em conta que a emergência do conhecimento científico confere prioridade à qualidade na prática clínica multicultural, sugerimos que futuramente se investigue a perceção dos diferentes profissionais de saúde perante a necessidade de construir competências culturais para cuidar de imigrantes, numa equipa multidisciplinar.

\section{Referências Bibliográficas}

Anes, C. M. L. S. (2006). Imigrantes em Portugal: Filhos ou enteados de uma nova nação? (Dissertação de Mestrado não publicada). Universidade Aberta, Lisboa, Portugal.

Bronfenbrenner, U. (2002). A ecologia do desenvolvimento humano: Experimentos naturais e planejados. (M. A. V. Veronese, Trad.). Porto Alegre, Brasil: Artmed. (Obra original publicada em 1994).

Campinha-Bacote, J. (2011). Delivering patient-centered care in the midst of a cultural conflict: The role of cultural competence. The Online Journal of Issues in Nursing, 16(2), $1-8$.

Durand, C., \& Blais, A. (2003). A medida. In B. Gauthier (Ed.), Investigação social: Da problemática à colbeita de dados (3 ed., pp.175-200). (N. Salgueiro, Trad.) Loures, Portugal: Lusociência. (Obra original publicada em 2000).

Flick, U. (2005). Métodos qualitativos na investigação científica. (A. M. Parreira, Trad.). Lisboa, Portugal: Monitor. (Obra original publicada em 2002).

Fonseca, M. L., Silva, S., Esteves, A., \& McGarrigle, J. (2009). Mighealthnet - Relatório sobre o estado da arte em Portugal. Lisboa, Portugal: Universidade de Lisboa, Centro de Estudos Geográficos.

Geoffrion, P. (2003). O grupo de discussão. In B. Gauthier (Ed.), Investigação social: Da problemática à colbeita de dados (3a ed., pp. 319-344). (N. Salgueiro \& R. P. Salgueiro, Trads.). Loures, Portugal: Lusociência. (Obra original publicada em 2000).

Leininger, M. M. (1994). Nursing and antropology: Two worlds to blend. Ohio, $\mathrm{OH}$ : Greyden.

Machado, M. C., Pereira, F., \& Machaqueiro, S. (2010). Approaches to migrant health in Portugal. Eurohealth, 16(1), 30-31. Recuperado de http://www.lse.ac.uk/ LSEHealthAndSocialCare/pdf/eurohealth/VOL16No1/ Machado.pdf 
Meleis, A. I. (2010). Role insufficiency and role supplementation: A conceptual framework. In A. I. Meleis (Ed.), Transitions theory: Middle-range and situation-specific theories in nursing research and practice (pp. 13-24). New York, NY: Springer.

Ordem dos Enfermeiros. Conselho de Enfermagem. (2003). Competências do enfermeiro de cuidados gerais. Lisboa, Portugal: Autor.

Pereira, M. A. D. A. (2008). A comunidade chinesa imigrante (Dissertação de Mestrado não publicada). Universidade Aberta, Lisboa, Portugal.

Purnell, L. (2011). Models and theories focused on culture. In J. B. Butts \& K. L. Rick (Eds.), Philosophies and theories for advanced nursing practice (pp. 225-568). Hattiesburg, MS: Jones \& Bartlett Learning.

Serrano, M. T. P., Costa, A. S. M. C. C., \& Costa, N. M. V. N. (2011). Cuidar em enfermagem: Como desenvolver a(s) competência(s). Revista de Enfermagem Referência, 3(3), 15-23.
Serviço de Estrangeiros e Fronteiras. Departamento de Planeamento e Formação, Núcleo de Planeamento. (2012). Relatório de imigração, fronteiras e asilo-2011. Lisboa, Portugal: Autor.

Skott, C., \& Lundgren, S. M. (2009). Complexity and contradiction: Home care in a multicultural area. Nursing Inquiry, 16(3), 223-231. doi:10.111/j.1440-1800.2009.00454.x

Spradley, J. P. (1980). Participant observation. Orlando, FL: Library of Congress Cataloging in Publication Data.

Streubert, H. J., \& Carpenter, D. R. (2013). Investigação qualitativa em enfermagem: Avançando o imperativo bumanista ( $5^{\mathrm{a}}$ ed). (A. P. S. S. Espada, Trad.). Loures, Portugal: Lusociência. (Obra original publicada em 1999).

Vega, A. (2010). Soignants/soignés: Pour une approche anthropologique des soins infirmiers. Belgique, Bruxelles: De Boeck. 
